\title{
Peronospora belbahrii causes downy mildew on Ocimum basilicum in Brazil
}

\author{
André Luis Silva ${ }^{1} \cdot$ Paloma Stefany Correa Mansur ${ }^{1} \cdot$ Robert Weingart Barreto $^{1} \cdot$ Sara Salcedo-Sarmiento $^{1}$ (D)
}

Received: 28 July 2019 / Accepted: 22 November 2019/Published online: 6 December 2019

(C) Australasian Plant Pathology Society Inc. 2019

\begin{abstract}
Downy mildew, caused by Peronospora belbahrii, is recorded for the first time in Brazil on sweet basil (Ocimum basilicum). A study of the morphological and molecular features led to its identification. Pathogenicity of $P$. belbahrii to sweet basil was tested and confirmed.
\end{abstract}

Keywords Culinary herb · Ocimum basilicum $\cdot$ Oomycete $\cdot$ Peronosporaceae $\cdot$ Vegetable

Sweet basil (Ocimum basilicum) is an important aromatic crop and source of essential oils, broadly cultivated worldwide (Pandiri and Moni 2018) including in Brazil (Vieira and Simon 2000). Despite its economic relevance, little has been published on fungal diseases attacking this crop in Brazil. A number of incomplete records (conference abstracts - with no complete fungus description, molecular information, demonstration of pathogenicity or even indication of voucher specimens or cultures) were listed in Mendes and Urben (2019).

Since November 2017, sweet basil (O. basilicum cv. Genovese) plants grown in a plot at the plant disease garden (Infectarium) of the Departamento de Fitopatologia (Universidade Federal de Viçosa, state of Minas Gerais, Brazil) have been observed bearing typical downy mildew symptoms on their leaves. Elongated intervenal chlorotic lesions developed adaxially, accompanied with a grey efflorescence abaxially and progressed towards complete necrosis of infected leaves. This led to intense defoliation and, sometimes, to plant death. A representative sample of infected leaves was dried in a plant press and deposited in the local herbarium (acc. Number:VIC 47166).

Sporangiophores and sporangia forming on the lower leaf surface were scraped with a scalpel and material was mounted

Sara Salcedo-Sarmiento taphrina10@gmail.com

1 Departamento de Fitopatologia, Universidade Federal de Viçosa, Viçosa, Minas Gerais 36570-900, Brazil on lactophenol and observed under a light microscope (Olympus BX53) fitted with differential interference contrast (DIC) illumination and connected to a Olympus Q-color 5 ${ }^{\mathrm{TM}}$ camera. The downy mildew pathogen had the following morphology: conidiophores arising from stromata, erect, firstly cylindrical then branching dichotomously three to five times, 200-835 × 8-16 $\mu \mathrm{m}$, hyaline, smooth (Fig. 1E); conidia, ovoid to subglobose $23-34 \times 21-28 \mu \mathrm{m}$, light brown (Fig. 1F). This morphology was recognised as almost identical to that described for Peronospora belbahrii by Thines et al. (2009).

In order to futher confirm the morphology-based identification, a sequence analysis of the cytochrome oxidase subunit 2 ( $\operatorname{cox} 2)$ region was conducted. Genomic DNA was extracted from conidia harvested from colonies on the plant tissue with the Wizard Genomic DNA Purification Kit (Promega) by following the manufacturer's instructions. The partial cox 2 region was amplified using the primers peronosporomycete forward (5'-GGCA AATGGGTTTTCAAGATCC-3') and reverse (5'C CATGAT TA ATA C C A CAAAT T TCA C TAC-3') (Hudspeth et al. 2000) and sequencing was performed by Macrogen (Seoul, Korea). The consensus sequence was deposited in GenBank (accession No. MN418242). BLASTn analysis in the NCBI database revealed $100 \%$ similarity to accession No. KJ654229 of $P$. belbahrii from $O$. basilicum. The identity of the oomycete involved on downy mildew of sweet basil in Brazil was confirmed as Peronospora belbahrii by comparison with the published data (Belbahri et al. 2005; Thines et al. 2009). 
Fig. 1 Peronospora belbahrii on Ocimum basilicum. (A-B)

diseased plants with internerval chlorotic lesions on leaves. (C) abaxial grayish downy mildew colonies. (D-F) Sporangia and sporangiophores of $P$. belbahrii. Bars $=50 \mu \mathrm{m}(\mathrm{D}-\mathrm{E})$ and $20 \mu \mathrm{m}$ (F)
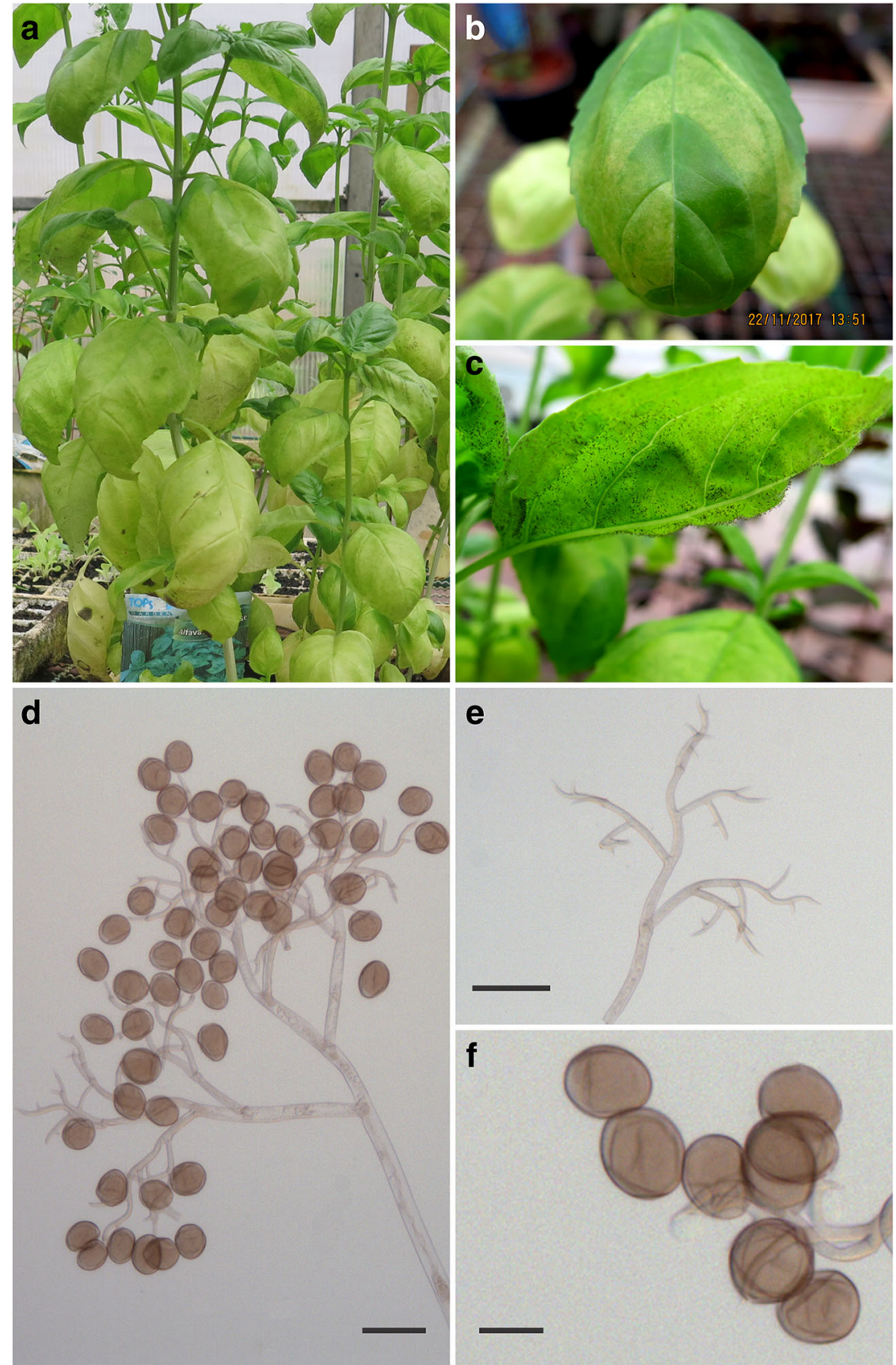

e
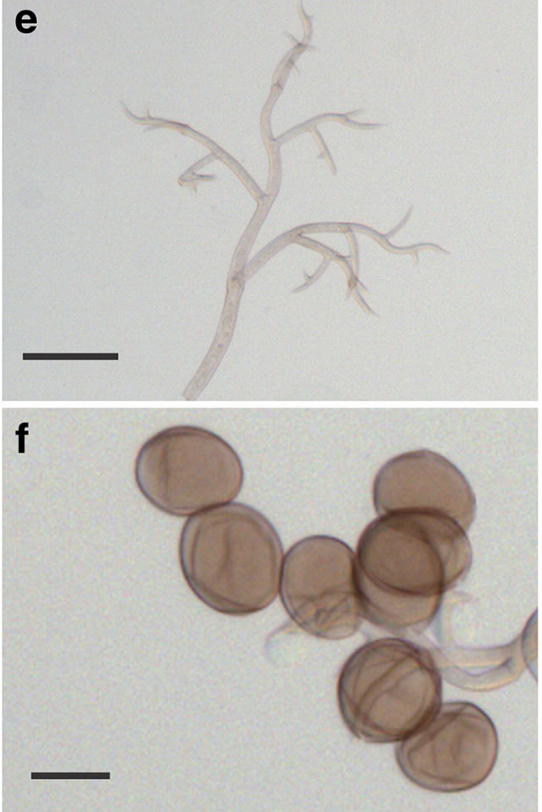

In order to confirm the pathogenicity of $P$. belbahrii to sweet basil, leaves of healthy two month potted seedlings of sweet basil cv. Genovese were inoculated with a suspension containing $1 \times 10^{5}$ conidia/ml (obtained by scarping sporulating lesions of naturally infected plants from the field with a scapel and suspending the conidia in sterile distilled water). Six healthy seedlings were sprayed until runoff, whereas two separate control seedlings were sprayed with distilled water. All plants were placed in a dew chamber for $48 \mathrm{~h}$ and then transferred to greenhouse bench at $25 \pm 2{ }^{\circ} \mathrm{C}$ and observed daily. Typical symptoms of downy mildew were observed on leaves 8 to 10 days after inoculation. Control plants remained healthy. Examinations of the morphology of the pathogen on diseased test-plants confirmed it as P. belbahrii.

The oomycete causing downy mildew of sweet basil was described as Peronospora belbahrii by Thines et al. (2009), but, according to Choi et al. (2016) this disease grew in importance - becoming the most destructive for sweet basil in countries Asia, Africa, Europe and the Americas - only during the past decade. Several records are listed by Farr and Rossman 2019, including recently in China (Hu et al. 2018). This is the first report of this sweet basil disease and of Peronospora belbahrii in Brazil. 


\section{References}

Belbahri L, Calmin G, Pawloski J, Lefort F (2005) Phylogenetic analysis and real time PCR detection of presumably undescribed Peronospora species on sweet basil and sage. Mycol Res 109: 1276-1287

Choi YJ, Choi IY, Lee KJ, Shin HD (2016) First report of downy mildew caused by Peronospora belbahrii on sweet basil (Ocimum basilicum) in Korea. Plant Dis 100:2335

Farr DF, Rossman AY (2019) Fungal database, U.S. National Fungus Colletions, ARS, USDA. https://nt.ars-grin.gov/fungaldatabases/ accessed 29th mar 2019

Hu B, Li Z, Hu H, Zheng J, Diao Y (2018) Outbreak of downy mildew caused by Peronospora belbahrii on Ocimum basilicum var. polosum in China. New Disease Reports 37:1
Hudspeth DSS, Nadler SA, Hudspeth MES (2000) A cox 2 molecular phylogeny of the Peronosporomycetes. Mycologia 92:674-684

Mendes MAS, Urben AF (2019) Fungos relatados em plantas no Brasil, Laboratório de Quarentena Vegetal. Embrapa Recursos Genéticos e Biotecnologia, Brasília, DF

Pandiri I, Moni A (2018) Ocimum herb species: a potential treatment strategy for diabetic kidney disease. Journal of Advanced Biotechnology and Experimental Therapeutics 1:88-91

Thines M, Telle S, Ploch S, Rungel S (2009) Identify of the downy mildew pathogens on sage, basil and coleus, with implications for quarantine measures. Mycol Res 113:532-540

Vieira RF, Simon JE (2000) Chemical characterisation of basil (Ocimum basilicum L.) found in markets and used in traditional medicine in Brazil. Econ Bot 54:207-216 